expérimentales de façon précise et constante qui avait masqué les phénomènes. Un exemple classique est donné par la résistance des solides immergés dans l'air à l'écoulement de l'air : On a longlemps cru que cette résistance avait une valeur assez forte, parce que pour obtenir des filets parallèles, on faisait préalablement passer l'air à travers une grille, laquelle provoquait un régime tourbillonnaire. Or les pylônes des lignes de distribution sont calculés en partant de cette valeur vraisemblablement exagérée de la résistance. La recherche précise intéresserait donc particulièrement Ies électriciens, et tous ceux qui s'occupent de houille blanche.

Or, d'une façon générale, Loules ces recherches sur les fluides ne sont possibles qu'avec un outillage puissant (Un jel de $10 \mathrm{~cm}$ de diamc̀tre, animé d'une vitesse de $80 \mathrm{~m}: \mathrm{sec}$

$$
\left[\frac{\mathrm{WD}}{\mathrm{y}}=6,4 \times 10^{6}\right]
$$

exige une puissance de plus de 2000 chevaux). Elles ne peuvent donc être poursuivies qu'en usine. Mais elles réclament aussi une connaissance parfaite des travaux antérieurs sur la question : Il ne s'agit pas d'avoir des nombres, il faut pouvoir les interpréter; il ne faut pas non plus ercommencer les expériences déjà faites. C'est donc par une collaboration incessante du laboratoire et de l'usine que les hydrauliciens pourront atteindre les hauts rendements auxquels, par la même collaboration, sont arrivés les constructeurs de matériel électrique.

\title{
Débit des Déversoirs à contraction latérale.
}

\author{
(Expériences de M. HÉGLY)
}

Tous les hydrauliciens de langue française connaissent la formule de Bazin pour l'évaluation du débit qui passe sur un déversoir en mince paroi, el sans contraction latérale :

$$
\mathrm{Q}=\mu \mathrm{mlh} V \overline{2 g h}
$$

dans larquelle

$$
u=0,405+\frac{0,003}{h}
$$

$$
m=1+0,55\left(\frac{h}{h+p}\right)^{2}
$$

$h$ étant la charge sur ce déversoir dont le seuil de largeur $l$ est à une hauteur $p$ au-dessus du fond du canal au travers duquel il est établi.

Maśs cette formule, qui permet d'évaluer le débit avec précision lorsqui on s'entoure de toutes les précautions nécessaires, n'est rigoureusement applicable que lorsque le déversoir est bien sans contractiou latérale, et avec une nappe libre, c'est-à-dire avec admission de la pression atmosphérique sous la nappe. Pour que cette double condition soit remplie, il faut, soit ménager un élargissement brusque du canal au débouché du déversoir, soit créer un rétrécissement artificiel du canal immédiatement à l'avant du dit déversoir.

Pour se dispenser de celte sujétion, on se contente souvent de donner au déversoir une largeur un peu inférieure à celle du canal. Il se produit alors une contraction latérale sur chacun des côtés, ce qui a pour effet de diminuer dans une certaine mesure la section d'écoulement. Pour en tenir compte, il faut modifier quelque peu la formule Bazin. Parmi les divers modes de correction jusqu'ici préconisés, l'un des plus fréquemment employés consiste à remplacer, comme dans la formule américaine de Francis, la largeur $l$ réelle du déversoir par une largeur tictive $l-\frac{n}{10} h$, soit $l-0,2 h$ dans le cas ordinaire, $n$ étant le nombre des contractions.

M. HÉgry, aujourd'hui ingénieur en chef des Ponts et Chaussées, qui fut, au début de sa carrière, le collaborateur de Bazin lorsque cet éminent hydraulicien procédait à ses expériences sur la rigole du canal de Bourgogne, près de Dijon, profitant de l'installation de déversoirs de jauge sur les rigoles d'alimentation du canal de la Marne à la Saône, a continué les expériences de Bazin, en les étendant au cas du déversoir à contraction latérale. Ces expériences furent exécutées sur la rigole du Moulin-Rouge, près de Langres, et M. Hégly en a donné un compte rendu détaillé dans un mémoire inséré dans le tome VI (novembre-décembre) des Annales des Ponts et Chaussées, de 1921.

Le procédé d'expérimentation adopté fut celui-là même donl se servit Bazin. Nous rappellerons qu'il consiste à faire passer dans la rigole d'expérience un certain débit (dont il n'est pas nécessaire d'ailleurs de connaître a priori la valeur) et à noter en même temps, lorsque le régime uniforme est établi, les charges sur deux déversoirs montés in tandem à la suite l'un de l'autre. L'un de ces déversoirs, préalablement taré, de manière à ce que son coeffjcient de débit soit exactement connu, sert de témoin tändis que l'autre déversoir fonctionne comme appareil à étudier

Les déversoirs soumis à l'expérience dans la rigole du Moulin. Rouge, dont la largeur avait été amenée à $2 \mathrm{~m}$. sur une longueur de $10 \mathrm{~m}$, furent disposés de façon que leur milieu correspondît exactement avec celui de la rigole de manière à ce que l'effet do contraction latérale fut le même des deux côtés. Ils étaient formés d'un panneau de bois surmonté d'une plaque en tôle d'acier dans laquelle une ouverture rectangulaire fut ménagée. La mince paroi était réalisée par un biseautage des bords de l'ouverture du côté aval de la plaque. La largeur $l$ de l'échancrure donnant passage à la lame déversante fut successivement de $1 \mathrm{~m} .80$, de $1 \mathrm{~m} .60$, de $1 \mathrm{~m}$. 20, de $0 \mathrm{~m}$. 80 et de $0 \mathrm{~m}$. 40. La hauteur de la base de l'échancrure au-dessus du fond de la rigole fut de $0 \mathrm{~m} .814$ dans une moitié des expériences, et de $0 \mathrm{~m}$. 412 dans l'aulre moitié.

L'examen des surfaces de la lame déversante a montré que la surface supérieure n'a plus la même régularité que celle des nappes sans contraction latérale. Deux sillons apparaissent aux extrémités, et des stries prennent naissance en amont de chaque bord vertical pour aller se prolonger en aval. Pour ce qui est de la surface inférieure, elle parâ̂t aussi nette que celle des veines sans contraction la térale, et le profil en long de cette surface inférieure ne paraît présenter aucune différence avec celui qu'a relevé Bazin sur les veines de ce genre. La' même régularité apparaît sur les faces latérales de la veine contractée. 
Il a été fait dix séries d'expériences de manière à étudicr l'action combinée des cinq largeurs différentes $l$ du déversoir avec les deux hauteurs $p$ au-dessus du fond du canal. Les débits utilisés ont varié de 20 litres par seconde, pour les faibles charges de la plus petite largeur, à 850 litres pour les fortes charges de la plus grande largeur.

De la discussion des résultats obtenus dans ces six séries d'expériences, M. Hégly en a déduit, pour $m$ et $\mu$, les expressions suivantes, dans lesquelles $L$ représente la largeur du canal ( $2 \mathrm{~m}$. au Moulin-Rouge).

$$
\begin{gathered}
\mu_{1}=0,405+\frac{0,0027}{h}-0,030 \frac{\mathrm{L}-l}{\mathrm{~L}} \\
m_{1}=1+0,55 \frac{\omega^{2}}{\Omega^{2}}=1+0,55 \frac{l^{2}}{\mathrm{~L}^{2}}\left(\frac{h}{h+p}\right)^{2}
\end{gathered}
$$

Le nouveau coefficient $m$ dont le rôle est de tenir compte de la vitesse d'arrivée de l'eau, peut se déduire directement de considérations théoriques. $\Omega$ y représente la section d'écoulement dans le canal, tandis que w représente la section brute d'écoulement sur le déversoir, mesurée au-dessous du plan d'eau amont.

Pour le coefficient $\mu$, la différence avec celui de Bazin provient surtout de son dernier terme qui intervient pour tenir compte des contractions latérales.

La diminution du débit provoquée par les contractions laté rales est donc égale à $0,030 \frac{\mathrm{L}-l}{\mathrm{~L}} m h / \sqrt{2 g h}$. et lee choses se passent comme si la largeur $l$ du déversoir était diminuée de la quantité $\frac{0,030(L-l)}{0,405 h+0,0027} \cdot \frac{l}{L} h$.

M. Hégly propose en outre, et cela d'une manière générale, qu'il y ait ou non contraction, de remplacer le terme $\frac{0,003}{h}$ de Bazin par le terme $\frac{0,0027}{h}$ qui semble mieux tenir compte des résultats observés avec de faibles charges.

M. Hégly a comparé les valeurs du coefficient de débit $\mathrm{C}=m_{\mu}$, que donne cette formule, avec ceux observés et publiés précédemment par divers expérimentateurs, et notamment par Castel, Lesbros, Poncet et Lesbros en France ; par Fteley et Sterns, Francis et Hamilton-Smith en Amérique, et par Freese en Allemagne. De cette comparaison, il s'en dégage une très grande concordance car, sauf pour les très petites charges, la différence est inférieure $a_{* t}^{\pi} u n$ pour cent.

M. Hégly a également effectué six autres séries d'expérience correspondant au cas de déversoirs à deux ouvertures de $0 \mathrm{~m} .40$, ou de $0 \mathrm{~m}$. 80 de largeur, ainsi qu'à celui de déversoirs à trois ouvertures de $0 \mathrm{~m}$. 40, symétriquement placés par rapport à l'axe de la rigole. Il a constaté que la formule proposée convenait encore parfaitement.

M. Hégly s'est préoccupé de ce que devenait la valeur du coefficient $p$ pour le cas d'un déversoir établi sur la rive d'un canal ou sur le bord d'un réservoir, cas pour lequel $L$ devient infiniment grand devant $l$. Selon lui, cetle valeur de $p$ serait assez exactement représentée par l'expression

$$
\mu_{2}=0,405+\frac{0,0027}{h}-\frac{0,033}{1+l}
$$

Toulefois, dans le cas d'un canal où l'eau arriverail avec unc vitesse assez grande, le débit calculé avec la formule ci-dessus ne serait plus qu'approché, car il est évident que l'écoulement ne se fait plus de la même façon lorsque l'eau arrive latéralcment que Iorsqu'elle arrive longitudinalement.

II est bien entendu que la valeur de $\mu$ donnée ci-dessus ne s'applique qu'au cas d'un déversoir en mince paroi. Dans lc cas habituel d'un déversoir à seuil épais, il faudrait en outre faire intervenir la correction indiquée par Bazin en fonction de l'épaisseur du seuil.

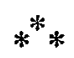

M. Hégly a terminé ses expériences du Moulin-Rouge par unc recherche du coefficient de débit de deux autres types de déversoirs, savoir le déversoir à section triangulaire avec angle droit, et le déversoir à section semi-circulaire.

Ces déversoirs, de même hauteur du seuil $p$ au-dessus du fond du canal que ceux précédemment étudiés, étaient constitués par une tôle présentant une échancrure appropriée : triangulaire de $0 \mathrm{~m} .50$ de haut sur $1 \mathrm{~m}$. de largeur au sommet, ou bien semicirculaire de $0 \mathrm{~m}$. 50 de rayon. La largeur $L$ du canal étant toujours de 2 mètres.

Pour le calcul des coefficients de débit de ces types de déversoirs, M. Hégly estime que, tant que (1) reste très petit devant $\Omega$, comme c'était le cas pour ses expériences, le coefficient $m$ peut être représenté simplement par $m_{3}=1+\frac{\omega^{2}}{\left(2^{2}\right.}$.

Pour ce qui est du coefficient $\mu$, M. Hégly propose les expressions :

$\mu_{3}=0,310+\frac{0,002}{h}$ pour le déversoir triangulaire;
$\mu_{3}=0,350+\frac{0,002}{h}$ pour le déversoir semi-circulaire.

La formule Thomson, qui est généralement employée pour le déversoir triangulaire, utilise pour le coefficient de débitc $=m_{\text {, }}$ la valeur $c=0,328$ (avec le système métrique). Dans les expériences de M. Hégly, cette valeur ne correspond qu'au cas où $h$ est plus petit que $200 \mathrm{~mm}$. Au-dessus de $200 \mathrm{~m} / \mathrm{m}$ la valeur trouvée pour $c$, qui varie peu, est voisine de $c=0,315$.

Cette différence des valeurs de $c$ peut provenir de la différence de la largeur des canaux d'amenée dans les deux cas, ce qui aurait pour effet de déterminer des vitesses d'arrivée inégales.

H. B. 